\title{
Comparative transcriptomic analysis reveals the cold acclimation during chilling stress in sensitive and resistant passion fruit (Passiflora edulis) cultivars
}

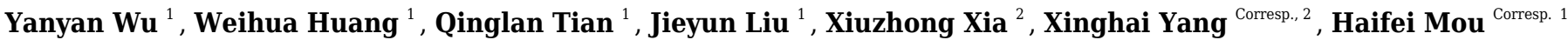 \\ 1 Biotechnology Research Institute, Guangxi Academy of Agricultural Sciences, Nanning, Guangxi, CHINA \\ 2 Rice Research Institute, Guangxi Academy of Agricultural Sciences, Nanning, Guangxi, CHINA \\ Corresponding Authors: Xinghai Yang, Haifei Mou \\ Email address: yangxinghai888@gxaas.net, mhf@gxaas.net
}

Chilling stress (CS) is an important limiting factor for the growth and development of passion fruit (Passiflora edulis) in winter in South China. However, little is known about how the passion fruit responds and adapts to CS. In this study, we performed transcriptome sequencing of cold-susceptible cultivar Huangjinguo (HJG) and cold-tolerant cultivar Tainong 1 (TN1) under normal temperature (NT) and CS conditions, and a total of 47,353 unigenes were obtained by 7 databases. Using differentially expressed unigenes (DEGs) analysis, 3,248 and 4,340 DEGs were identified at two stages, respectively. The Gene Ontology (GO) enrichment analysis showed that the DEGs were mainly related to phosphorylation, membrane protein, and catalytic activity. In Kyoto Encyclopedia of Genes and Genomes (KEGG) pathway, the unigenes of plant-pathogen interaction, plant hormone signal transduction and fatty acid metabolism were enriched. Then, the 12,471 filtered unigenes were clustered into eight co-expression modules, and two modules were correlated with CS. In this two modules, 32 hub unigenes were obtained. Furthermore, the unigenes related to CS were validated using quantitative real-time PCR (RT-qPCR). This work showed that the expression levels of CS-related unigenes were very different in two passion fruit cultivars. The results provide information for the development of passion fruit with increased chilling tolerance. 
1 Title: Comparative transcriptomic analysis reveals the cold acclimation

2 during chilling stress in sensitive and resistant passion fruit (Passiflora edulis)

3 cultivars

4 Yanyan $\mathrm{Wu}^{1}$, Weihua Huang ${ }^{1}$, Qinglan $\operatorname{Tian}^{1}$, Jieyun Liu ${ }^{1}$, Xiuzhong Xia ${ }^{2}$, Xinghai Yang ${ }^{2 *}$,

5 Haifei Mou ${ }^{1 *}$

6 1Biotechnology Research Institute, Guangxi Academy of Agricultural Sciences, Nanning,

7 Guangxi 530007, China

8 2Rice Research Institute, Guangxi Academy of Agricultural Sciences, Nanning, Guangxi 530007,

9 China

10 *Corresponding author: Xinghai Yang; Department/Institute: Rice Research Institute,

Guangxi Academy of Agricultural Sciences; Address: 174 East Daxue Road, Nanning, Guangxi 530007, Chian; E-mail: yangxinghai514@163.com; Tel: +867713244040; ORCID ID: https://orcid.org/0000-0002-3476-2578.

*Co-corresponding author: Haifei Mou; Department/Institute: Biotechnology Research Institute, Guangxi Academy of Agricultural Sciences; Address: 174 East Daxue Road, Nanning, Guangxi 530007, China; E-mail: mhf@gxaas.net; Tel: +86771 3243531 


\section{Abstract}

Chilling stress (CS) is an important limiting factor for the growth and development of passion fruit (Passiflora edulis) in winter in South China. However, little is known about how the passion fruit responds and adapts to CS. In this study, we performed transcriptome sequencing of cold-susceptible cultivar Huangjinguo (HJG) and cold-tolerant cultivar Tainong 1 (TN1) under normal temperature (NT) and CS conditions, and a total of 47,353 unigenes were obtained by 7 databases. Using differentially expressed unigenes (DEGs) analysis, 3,248 and 4,340 DEGs were identified at two stages, respectively. The Gene Ontology (GO) enrichment analysis showed that the DEGs were mainly related to phosphorylation, membrane protein, and catalytic activity. In Kyoto Encyclopedia of Genes and Genomes (KEGG) pathway, the unigenes of plant-pathogen interaction, plant hormone signal transduction and fatty acid metabolism were enriched. Then, the 12,471 filtered unigenes were clustered into eight co-expression modules, and two modules were correlated with CS. In this two modules, 32 hub unigenes were obtained. Furthermore, the unigenes related to CS were validated using quantitative real-time PCR (RT-qPCR). This work showed that the expression levels of CS-related unigenes were very different in two passion fruit cultivars. The results provide information for the development of passion fruit with increased chilling tolerance.

Key words Passion fruit, Chilling stres, RNA-seq, WGCNA, Hub genes, RT-qPCR

\section{Introduction}

Passion fruit is a tropical and subtropical fruit tree that is widely planted in South China and its fruit has an aromatic smell and high nutritional values. But passion fruit is susceptible to cold stress in winter (Liu et al. 2017A), which can cause large economic loss.

Cold stress is one of the limiting factors for plant growth and development (Shi et al. 2018). In plants, cold stress is classified into $\mathrm{CS}\left(0-15^{\circ} \mathrm{C}\right)$ and freezing stress $\left(<0{ }^{\circ} \mathrm{C}\right)$ (Yadav 2010; Shi et al. 2018). The cold environment can cause changes in the structure and activity of proteins in 
54

55

56

57

58

59

60

plant cells, leading to altered enzymatic reactions such as photosynthesis and respiration, and eventually leading to symptoms such as wilting and yellowing of plant leaves (Hendrickson et al. 2006). When plants are in reproductive growth, cold stress can cause damage of the plant reproductive organs, and the seed setting rate will be significantly reduced, which will eventually affect crop yields and cause major losses to agricultural production. Plants can gain resistance to low temperature, and this process is called cold acclimation. The cold acclimation of plants includes changes in a variety of intracellular physiological and biochemical processes. The most significant changes include the instantaneous increase of calcium ion concentration (Carpaneto et al. 2007), growth cessation, decrease in tissue water content affects on plant hormones abscisic acid (ABA), brassinolide (BR), and gibberellin (GA), cause fatty acid unsaturation and lipid peroxidation (Hara et al. 2003), changes in phospholipid composition (Webb et al. 1994), and penetrating substance such as proline, betaine and soluble sugar (Krasensky et al. 2012). The molecular mechanism of cold acclimation is that non-freezing low temperature can induce plants to express a series of cold response proteins to help plants resist freezing at low temperature. Inducer of $\mathrm{CBF}$ expression (ICE )- C-repeat binding factors (CBF )- cold-regulated proteins (COR) is thought to be one of the most important defense pathways in plants against cold stress (Shi et al. 2018). CBF can regulate the expression of COR by binding to the Crepeat/dehydration-responsive element (CRT/DRE) sequence that resides in the promoter region of the COR gene (Stockinger et al. 1997; Liu et al. 1998). ICE1 is located upstream of CBF, and it is a MYC-like bHLH type transcription factor, which can bind to the recognition site of the $C B F 3$ promoter and regulate its expression (Chinnusamy et al. 2003) Moreover, some CBFindependent transcription factors are involved in modulating COR expression, and various transcription factors, including CAMTA3 (Doherty et al. 2009), ZAT12 (Vogel et al. 2005), and HY15 (Catalá et al. 2011) can regulate the expression of CBFs. Protein phosphorylation also plays an important role in regulating the response of plants to low temperature (Mann m 2003), and mitogen-activated protein kinase (MAPK) as an important element in signal transmission (Zhao et al. 2017A). 
81

82

Guangxi belongs to a tropical and subtropical monsoon climate. The coldest month in January has an average daily temperature of $5.5^{\circ} \mathrm{C}$ to $15.2^{\circ} \mathrm{C}$. The continuous low temperature in winter affects the growth of passion fruit. However, no systematic study on the CS of passion fruit has been reported. In this study, the RNA-seq data was used to analyze gene expression during CS in passion fruit cultivars HJG and TN1. The main aims are to (i) analyze the gene expression profile of passion fruit during CS, (ii) explore the functions of DEGs, (iii) construct regulation network of the interactions of chilling tolerance genes of passion fruit, and (iv) identify the hub genes that affect the CS of passion fruit.

\section{Materials and Methods}

Plant materials

HJG was introduced in the Bannahuangguo of Xishuangbanna Botanical Garden in Yunnan, and it is a cold-sensitive accession. TN1 comes from Taiwan, and it is a cold-resistant purple passion fruit. The cutting seedling heights ranged from 29 to $38 \mathrm{~cm}$, and the seedlings were transplanted in Nanning experimental field (Guangxi, China, $22.85^{\circ} \mathrm{N}, 108.26^{\circ} \mathrm{E}$ ) on May 25, 2019. The first sampling time was November 25,2019 , at 10 a.m., and the temperature was $25^{\circ} \mathrm{C}$. The second sampling time was January 18, 2020, at 10 a.m., and the temperature was $7{ }^{\circ} \mathrm{C}$. The fresh leaves of passion fruit were snap frozen in liquid nitrogen, and then stored in $-80^{\circ} \mathrm{C}$ freezer. Each sample had three biological replicates. Under NT condition, the three biological replicates of HJG, HJGA1, HJGA2 and HJGA3, were recorded as A1; the three biological replicates of TN1, TN1A1, TN1A2 and TN1A3 were recorded as A2. Under CS condition, three biological replicates of HJG, HJGB1, HJGB2 and HJGB3, were denoted as B1; three biological replicates of TN1, TN1B1, TN1B2 and TN1B3, were denoted as B2.

\section{RNA extraction, sequencing, assembly and annotation}

Total RNA was extracted with RNAprep Pure kit (Tiangen, Beijing, China) according to the manufacturer's instructions. Nanodrop2000 (Shimadzu, Japan) was used to detect the concentration and purity of the extracted RNA. Agarose gel electrophoresis was used to detect the integrity of the RNA, and Agilent 2100 (Agilent, America) was used to determine the RIN 
108

109

110

111

112

113

114

value. A single library requires $1 \mu \mathrm{g}$ of RNA, with a concentration of $\geq 50 \mathrm{ng} / \mu \mathrm{L}$, and OD260/280 between 1.8 and 2.2. Magnetic beads (Invitrogen, America) with Oligo (dT) was used to pair with the 3 ' poly A tail of eukaryotic mRNA, thus isolating mRNA from total RNA. Subsequent, reverse synthesis of cDNA was performed. These libraries above were sequenced using an Illumina NovaSeq 6000 sequencer (Illumina Inc., USA) and 150-bp paired-end reads were generated. In order to ensure the accuracy of subsequent analysis, the original sequencing data were filtered first to obtain clean data.

We used Trinity v2.8.6 (Haas et al. 2013) to splice the transcript fragments to obtain transcripts, and then used CD-HIT to cluster the transcript sequences to remove redundant sequences and get all the unigene sequence sets for the subsequent analysis. Bowtie 2 (Salzberg et al. 2012) was used to align the sequencing data to the reconstructed unigene sequence set, and the alignment file was mainly used for subsequent unigene quantification and differential expression analysis. The unigene sequences were compared with the NCBI non-redundant protein sequences (NR), Swiss-Prot, TrEMBL, KEGG, GO, Pfam, and EuKaryotic Orthologous Groups (KOG) databases using Basic Local Alignment Search Tool (BLAST). Finally, HMMER3 (Finn et al. 2011) was used to align the amino acid sequence of unigene with the Pfam database to obtain the annotation information of unigenes.

\section{Enrichment analysis of DEGs}

The read counts and transcripts per million reads (TPM) were calculated using RSEM (Li et al. 2011) and bowtie2 (Salzberg et al. 2012). The DEGs were identified through the software packages of Bioconductor 3.11-DESeq2 (Love et al. 2014). The screening threshold is false discovery rate $(\mathrm{FDR})<0.05$, and $\log 2$ fold change $(\mathrm{FC}$ (condition $2 /$ condition 1$)$ for a gene) $>1$ or $\log 2 \mathrm{FC}<-1$. The DEGs were classified, and GO and KEGG enrichment analysis were subsequently performed.

\section{Weighted gene co-expression network analysis}

We followed these steps below for weighted gene co-expression network analysis (WGCNA): (i) screening DEGs for WGCNA cluster analysis; (ii) calling the R package to cluster the DEGs; (iii) 
135

136

137

138

139

140

141

142

143

144

145

146

147

148

149

150

151

152

153

154

155

156

157

158

159

160

161

calling ggplot2 in the R package to draw the clustering heat map and histogram of each module; (iv) using the top GO terms to perform GO enrichment analysis on each module; (v) calling Fisher-test function in R for KEGG enrichment analysis; and (vi) using Cytoscape3.8.0 (Su et al. 2014) to draw network diagram. A signed network was constructed using the blockwiseModules function, with the following parameters: power $=14$, minModuleSize $=30$, mergeCutHeight $=$ 0.25 , corType $=$ pearson. When co-expressed genes are defined according to the abovementioned standards, each gene is assigned a module number and corresponding module color; otherwise the 'gray' module was used.

\section{Validation of the CS-related genes using RT-qPCR}

We selected 11 genes related to plant hormone signaling, fatty acid metabolism and plantpathogen interaction using GO and KEGG databases, and 4 hub genes in WGCNA for validation. The primers were designed using Primer3 (Table S1). Using HIS as the reference gene (Liu et al. 2017A), RT-qPCR was used to analyze the expression level of 15 genes in B1 and B2.

The identical RNA samples as RNA-seq experiments were used for RT-qPCR analysis. The detailed experimental method refers to $\mathrm{Wu}$ et al. (Wu et al. 2020). The relative gene expression level was calculated by reference to the $2^{-\Delta \Delta C t}$ method (Livak et al. 2001). All unigenes expression analysis were performed in triplicates. The values represented arithmetic averages of three replicates, and the data were expressed as a mean plus and minus standard deviation (mean $\pm \mathrm{SD})$.

\section{Statistical analysis}

CASAVA was used for base calling. Subsequently, we used SeqPrep for quality control of raw sequencing data. Pearson correlation coefficient is used to measure the correlation between samples. The package heatmap of $\mathrm{R}$ was used to prepare the correlation between samples and DEGs expression pattern clustering. Data of RT-qPCR was analyzed using Excel 2007. The figures were prepared using Origin 9.65.

Results

Quality control and assembly of passion fruit transcriptome sequences 
162 To compare gene expression profiles of the two passion fruit cultivars under NT and CS, 163 transcriptome sequencing and analysis were performed. After decontamination and adaptor 164 removal, 533,935,574 raw reads were obtained from 12 samples (NCBI accession number: 165 PRJNA634206), a total of $80.09 \mathrm{~Gb}$ clean reads and $6.67 \mathrm{~Gb}$ per sample. The Q30 base 166 percentage was $93.22 \%$ and GC content was $44.64 \%$ (Table 1 ).

167 The clean reads were assembled into transcripts using the Trinity in paired-end method, and 168211,874 transcripts were obtained (https://figshare.com/s/ef47208bf0d06c3d9bde). The CD-HIT 169 was then used to cluster the transcripts, which yielded 47,353 unigenes

170 (https://figshare.com/s/89666a7f0fe7d4df353e) with a mean length of 1,211 bp, N50 length of 2,368 bp, and N90 of $450 \mathrm{bp}$. Afterwards, Bowtie 2 was used to align the sequences of each

172 sample to the unigene sequence set, with an average alignment ratio of $77.89 \%$ (Table 2).

\section{Unigene function annotation}

174

The assembled unigenes were annotated to databases including the NR, Swiss-Prot, TrEMBL, KEGG, GO, Pfam, and KOG, to which $97.92 \%, 70.40 \%, 97.82 \%, 33.97 \%, 36.16 \%, 61.43 \%$, and $48.92 \%$ of unigenes were mapped, respectively. A total of 47,353 unigenes acquired annotation information (Table 3). The number of annotated unigenes in NR and TrEMBL was the largest, which were 46,369 and 46,323, respectively.

In the GO database, 17,123 unigenes were annotated and matched to three major categories: biological process (BP), cellular component (CC) and molecular function (MF). Enriched BP terms were mainly about "metabolic process" $(4,350)$ and "cellular process" $(2,191)$. Enriched CC terms were mainly about "membrane part" $(1,270)$ and "cell part" (890). Enriched MF terms were mainly about "binding" (7,367) and "catalytic activity" (5,715) (Fig. 1A).

In KOG database, 23,164 unigenes were annotated, which were clustered into 25 categories. The unigenes were mainly about "signal transduction mechanisms" $(2,439)$ and "posttranslational modification, protein turnover, chaperones" (2,138) (Fig. 1B). In KEGG database, 16,086 unigenes were annotated. According to the functions, these unigenes were enriched in 9 pathways. The enriched pathways were mainly about "metabolism" 
189

190

191

192

193

194

195

196

197

198

199

200

201

202

203

204

205

206

207

208

209

210

211

212

213

214

215

$(10,045)$ and "organismal systems" (4,505) (Fig. 1C).

\section{Comparative analysis of DEGs in two cultivars at two stages}

In order to gain insights on the adaptation of passion fruit to CS, the TPM method was used to analyze the gene expression levels in the two stages (Fig. S1). The correlation coefficient between the three biological replicates was 0.87 in HJGA, 0.98 in TN1A, 0.96 in HJGB, 0.99 in $\mathrm{TN} 1 \mathrm{~B}$, and the average correlation coefficient value was 0.95 (Fig. S2), indicating that the reproducibility of this study was good and the experimental results were reliable.

$$
\text { The software package DESeq2 was used to perform differential expression analysis of }
$$
unigenes. There were 3,248 and 4,340 DEGs at two stages, respectively. After, analysis of the DEGs for the two stages, we found that the DEGs between HJG and TN1 were increased by 33.6\% under CS condition (1,092), and 87.5\% (955) were up-regulated (Fig. 2A).

Cluster analysis of gene expression can intuitively reflect the level of gene expression and expression patterns in multiple samples. We used the DEGs to perform cluster analysis on A1 vs A2 (Fig. 2B) and B1 vs B2 (Fig. 2C). The results showed that the difference between the three biological replicates of each group was small, which again confirmed the rationality of sample selection.

\section{GO and KEGG pathway enrichment analysis of DEGs}

There were 1,182 up-regulated unigenes, and 2,066 down-regulated unigenes at stage A; and there were 2,137 up-regulated unigenes and 2,203 down-regulated unigenes at stage B.

GO enrichment analysis indicated that "metabolic process" (542), "oxidation-reduction process" (156), "protein phosphorylation” (92), “carbohydrate metabolic process"(73), "organic substance catabolic process" (40), and "catabolic process" (40), "extracellular region" (10), “apoplast” (8), "cell wall” (8), "external encapsulating structure” (8), "catalytic activity” (634), “transferase activity” (228), "oxidoreductase activity" (167), "metal ion binding" (146), "cation binding” (146), and "transition metal ion binding” were enriched at stage A (110) (Table S2). But "oxidation-reduction process" (187), "phosphate-containing compound metabolic process" (171), "phosphorus metabolic process" (171), "macromolecule modification" (170), "cellular 
216 protein modification process" (169), and "protein modification process" (169), "membrane"

217 (213), "intrinsic component of membrane" (99), and "integral component of membrane"

218 (97), “catalytic activity” (837), "transferase activity” (326), “cation binding” (202), “metal ion

219 binding” (201), "oxidoreductase activity" (198), "phosphotransferase activity", and "alcohol

220 group as acceptor" (165) were enriched at stage B (Table S3).

221 The GO terms in $\mathrm{A}(\mathrm{P}>0.05)$ were compared to $\mathrm{B}(\mathrm{P} \leq 0.05)$, and the unigenes were mainly

222 about "protein phosphorylation” (GO:0006468, 61) “phosphorylation” (GO:0016310, 61),

223 "response to stimulus" (35), "lipid metabolic process" (19), "response to chemical” (13),

224 "membrane" (73), "intrinsic component of membrane" (29), "integral component of membrane"

225 (28) "catalytic activity", "acting on a protein" (77), "transferase activity", "transferring

226 phosphorus-containing groups" (70), "kinase activity" (67), "phosphotransferase activity”,

227 "alcohol group as acceptor" (67), and "protein kinase activity" (62) (Table S4).

228 The KEGG pathway enrichment analysis can reveal the main metabolic pathways and signal 229 transduction pathways in which the DEGs were involved, and the prevailing pathways were as 230 follows: "ribosome" (42), "carbon metabolism" (39), "biosynthesis of amino acids" (30), "starch

231 and sucrose metabolism" (21), "and cysteine and methionine metabolism" (20) at stage A; "plant 232 hormone signal transduction" (31), "plant-pathogen interaction" (27), "fatty acid metabolism" 233 (21), "cysteine and methionine metabolism" (20) (Fig. 3A). The KEGG pathway in A (P>0.05) 234 were compared to B (P $\leq 0.05)$, and the unigenes were mainly about "plant-pathogen interaction" 235 (17), "plant hormone signal transduction" (14), and "fatty acid metabolism" (8) (Fig. 3B).

236

237

238

239

240

241

242

\section{WGCNA analysis}

After background correction and normalization of the unigenes expression data, we filtered out the abnormal and minor changed unigenes. Finally, we obtained 12,471 highly expressed unigenes (Table S5). In this study, when the soft threshold was 16 (Fig. S3), the gene topology matrix expression network was closest to the scale-free distribution. A gene cluster tree was constructed based on the correlation between genes, and each branch corresponded to a cluster of gene sets with highly correlated expression levels (Fig. S4a). 
According to the standard of mixed dynamic shear, the gene modules were classified and the eigenvector of each module was calculated. The modules close to each other were merged, and 8 co-expression modules were obtained (Fig. S4b). Each module used different colors to represent the clustered genes. The turquoise module had the most clustered genes $(4,171)$, the red module contained the fewest (81), and the grey module contained the unigenes that couldn't be included in any module.

The DEGs were used to draw the heat map of each module in the four sample groups. The brown and yellow modules showed less changes in differential unigenes between the early and late HJG, but showed larger changes in differential unigenes between early and late TN1 (Fig.4), which is consistent with the chilling resistance feature of TN1. Therefore, we selected the unigenes of these two modules for in-depth GO and KEGG pathway analysis.

In the brown module, the significant GO terms were "cellular macromolecule metabolic process", "phosphate-containing compound metabolic process", "phosphorus metabolic process", "protein phosphorylation", "stimulus", "transferase complex", "riboflavin synthase complex", "photosystem I reaction center", "photosystem I" "binding”, "metal ion binding”, "cation binding”, "phosphotransferase activity”, “alcohol group as acceptor”, "kinase activity” (Table S6). In the KEGG pathway analysis, the prevailing pathways were "plant hormone signal transduction", "MAPK signaling pathway", and "starch and sucrose metabolism" (Fig. 5A).

In the yellow module, the significant GO terms were "cellular process", "macromolecule modification", "phosphorus metabolic process", "cellular protein modification process", "protein modification process", "cell periphery", "photosystem", "photosynthetic membrane”, "thylakoid", "extracellular region", "3-deoxy-7-phosphoheptulonate synthase activity", “alkylbase DNA N-glycosylase activity", "DNA-3-methyladenine glycosylase activity”, "DNA N-glycosylase activity", and "method adenosyltransferase activity" (Table S7). The significantly enriched pathways included "biosynthesis of amino acids", "plant hormone signal transduction", "ABC transporters", "starch and sucrose metabolism", "folate biosynthesis" and "other pathways" that might be related to CS (Fig. 5B). 
270

271

272

273

274

275

276

277

278

279

280

281

282

283

284

285

286

287

288

289

290

291

292

293

294

295

296

The constructed network was visualized with the Cytoscape in the brown and yellow modules, and got 19 hub unigenes which mainly related to "MAPK signaling pathway", "plant hormone signal transduction", "starch and sucrose metabolism", "fatty acid biosynthesis" and "photosynthesis in the brown module" (Fig. 6A). In the yellow module, 13 hub unigenes were mainly related to "plant hormone signal transduction", "MAPK signaling pathway", "starch and sucrose metabolism", and "fatty acid degradation" (Fig. 6B).

\section{Validation of gene expression changes during chilling acclimation}

We used the RT-qPCR method to validate the expression level of 15 unigenes. The results showed that the RT-qPCR expression patterns of the 15 unigenes were consistent with RNA-seq analysis (Fig. 7, Table S8). RT-qPCR analysis showed that the 9 unigenes were $\geq 2$ or $\leq 0.5$ foldchange. Comparison with B1 and B2, TPM value of 12 unigenes were $\geq 2$ or $\leq 0.5$ fold-change. The results showed that seventy-five percent DEGs could be validated using RT-qPCR, and DEGs analysis were highly reliable.

\section{Discussions}

Low temperature is one of the main abiotic stresses that the plants are vulnerable to during their life cycle, and the response of plants to low temperature stress is a multi-factor synergistic process involving complex physiological and gene expression regulatory networks. With the development of molecular biology technology, researchers have cloned many low temperature related genes in Arabidopsis thaliana (Wang et al. 2019; Ding et al. 2018; Ye et al. 2019) and rice (Ma et al. 2015; Zhang et al. 2017A) . Passion fruit is a tropical and subtropical fruit tree and it is vulnerable to low temperature in winter. However, there are fewer studies on cold stress in passion fruit. In this study, the passion fruit cultivar of TN1 was identified, which has the characteristics of cold-tolerance.

Although the two cDNA libraries were constructed for transcriptome sequencing in passion fruit under CS condition (Liu et al. 2017A), we still know little about the cold tolerance of passion fruit. To reveal the expression pattern of CS-related genes in passion fruit, RNA-seq analysis were performed. Using database function annotation, we obtained 47,353 unigenes 
297

298

299

300

301

302

303

304

305

306

307

308

309

310

311

312

313

314

315

316

317

318

319

320

321

322

323

(https://figshare.com/s/89666a7f0fe7d4df353e). Based on RNA-seq data, the number of downregulated DEGs did not change much at two stages, but the number of up-regulated DEGs were 955, indicating that the up-regulation of DEGs maybe related to CS.

Protein phosphorylation is also a type of post-translational regulation during the cold

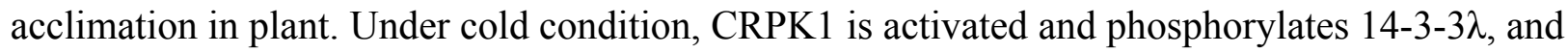

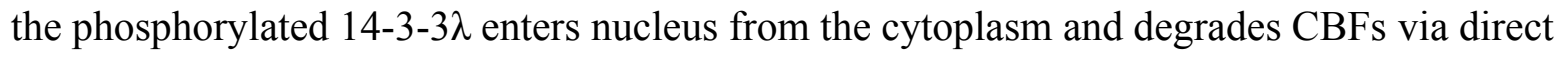
interaction in Arabidopsis thaliana (Liu et al. 2017B). Transcriptome sequencing revealed that 61 DEGs of phosphorylation were significantly up-regulated or down-regulated in the two stages, respectively (Table S4). Furthermore, the unigenes were mainly related to calcium-dependent protein kinase, serine/threonine-protein kinase, and CBL-interacting serine/threonine-protein kinase. In rice, calcium-dependent protein kinase gene OsCPK17 (Almadanim et al. 2018), OsCDPK7 (Saijo et al. 2000) and OsCPK24 (Liu et al. 2018B) all respond to low temperature. In a previous study, serine/threonine protein kinase responded to cold stress (Soto et al. 2002).

These results indicated that protein phosphorylation could play an important role in cold acclimation of passion fruit.

Mitogen-activated protein kinase (MAPK) plays an important role in signal transduction, and is also essential for regulating the cold response of plants. Under low temperature, the phosphorylation levels of MPK3, MPK4 and MPK6 were significantly increased (Zhao et al. 2017B); moreover, MPK3 and MPK6 could interact with ICE1 to participate in low temperature response (Li et al. 2017). Zhang et al. found that the phosphorylated OsICE1 could promote OSTPP1 transcription and induce the production of large amounts of trehalose, thereby improving the cold resistance of rice (Zhang et al. 2017B). Using WGCNA analysis, we found that MAPK signaling pathway significantly enriched in the brown module, which contained 7 DEGs (Fig. 5A). Moreover, the functional annotation of TRINITY_DN36339_c2_g1_i5 was mitogen-activated protein kinase kinase kinase 3. In rice, OsMKK6 and OsMPK3 constitute a moderately low-temperature signalling pathway and regulate cold stress tolerance (Xie et al. 2012). MKK2 induces the expression of COR genes to enhance the freezing tolerance of 
324

325

326

327

328

329

330

331

332

333

334

335

336

337

338

339

340

341

342

343

344

345

346

347

348

349

350

Arabidopsis thaliana (Teige et al. 2004).

In plants, hormones and cold signaling pathways are coordinated to better adapt to cold stress.

ABA is used as an important signal molecule and the most important stress signal in hormones, and it can mediate the signal transduction pathway to cold stress and increase the tolerance of cold stress (Yuan et al. 2018B). Auxin acts as a trigger in plant growth and development. In rice, ROC1 can regulate $C B F 1$, and auxin can affect ROC1 levels (Dou et al. 2016). In addition, BR, GA, JA, ethylene, CK, and melatonin play important regulatory roles in the ICE-CBF-COR pathway (Wang et al. 2017). In CS condition, we found 31 unigenes about plant hormone signal transduction (Fig. 3A). In WGCNA analysis, the pathway of plant hormone signal transduction was enriched in brown and yellow modules. These unigenes were annotated about aux, JA, ABA, and BR.

Plants use fatty acid dehydrogenase to regulate the increase of fatty acid unsaturation to improve the cold resistance (Upchurch 2008; He et al. 2015). The change of malondialdehyde content caused by lipid peroxidation is negatively correlated with plant cold resistance (Kim et al. 2011). In this study, the unigenes related to fatty acid metabolism and lipid metabolic process were identified (Fig. 3A, Fig. 6B). Among them, 16 unigenes were annotated as delta (12)-fattyacid desaturase (FAD2). In rice, $O s F A D 2$ is involved in fatty acid desaturation and maintenance of the membrane lipids balance in cells, and could improve the low temperature tolerance (Shi et al. 2012). Similarly, FAD2 could improve the salt tolerance during seed germination and early seedling growth (Zhang et al. 2012), but FAD 8 was strongly inducible by low temperature in Arabidopsis thaliana (Gibson et al. 1994). The results indicated that FAD2 could improve the CS of passion fruit.

In the process of cold acclimation in plants, the hydrolysis of starch is intensified and the content of soluble sugar increases. As a result, the freezing point of cell fluid is lowered and the excessive dehydration of cells is reduced (Krasensky j 2012; Yue et al. 2015). The analysis of pathway enriched by KEEG and WGCNA revealed starch and sucrose metabolism related to cold stress was enriched at stage B. Three DEGs were obtained at stage B compare to stage A, 
351

352

353

354

355

356

357

358

359

360

361

362

363

364

365

366

367

368

369

370

371

372

373

374

375

376

377

378

379

380

381

382

383

384

385

and these unigenes were annotated as beta-glucosidase and glucan endo-1,3-beta-glucosidase 3-

like genes.

\section{Conclusions}

In this study, we performed a comprehensive comparative transcriptome analysis between two passion fruit cultivars, to identify the gene expression level and analyze molecular mechanism of CS. This work showed that the unigenes of protein phosphorylation, MAPK signaling, plant hormones and fatty acid metabolism play important roles in the chilling tolerance between the two passion fruit cultivars. Furthermore, 32 hub unigenes were assigned to two modules, which could play a vital role in the chilling acclimation of passion fruit. In all, these findings provide a deepened understanding of the molecular mechanism of cold stress and could facilitate the genetic improvement of chilling tolerance in passion fruit.

\section{Acknowledgments}

The authors thank to Dr. Yinghua Pan for help providing data analysis suggestions.

\section{References}

Almadanim MC, Gonçalves NM, Rosa M, Alexandre BM, Cordeiro AM, Rodrigues M, Saibo N, Soares CM, Romão CV, Oliveira MM, Abreu IA. 2018. The rice cold-responsive calcium-dependent protein kinase OsCPK17 is regulated by alternative splicing and post-translational modifications. Biochim Biophys Acta Mol Cell Res 1865: 231-246.

Carpaneto A, Ivashikina N, Levchenko V, Krol E, Jeworutzki E, Zhu J, Hedrich H. 2007. Cold transiently activates calcium-permeable channels in Arabidopsis mesophyll cells. Plant Physiol 143: $487-$ 494.

Catalá R, Medina J, Salinas J. 2011. Integration of low temperature and light signaling during cold acclimation response in Arabidopsis. Proc Natl Acad Sci U S A 108: 16475-16480.

Chinnusamy V, Ohta M, Kanrar S, Lee BH, Hong X, Agarwal M, Zhu JK. 2003. ICE1: a regulator of cold-induced transcriptome and freezing tolerance in Arabidopsis. Genes Dev 17: 1034-1054.

Ding Y, Jia Y, Shi Y, Zhang X, Song C, Gong Z, Yang S. 2018. OST1-mediated BTF3L phosphorylation positively regulates CBFs during plant cold responses. EMBO J 37: e98228.

Doherty CJ, Van buskirk HA, Myers SJ, Thomashow MF. 2009. Roles for Arabidopsis camta transcription factors in cold-regulated gene expression and freezing tolerance. Plant Cell 21: 972-984.

Dou M, Cheng S, Zhao B, Xuan Y, Shao M. 2016. The indeterminate domain protein ROC1 regulates chilling tolerance via activation of DREB1B/CBF1 in rice. Int J Mol Sci 17: 233.

Finn RD, Clements J, Eddy SR. 2011. HMMER web server: interactive sequence similarity searching. Nucleic Acids Res 39: W29-W37.

Gibson S, Arondel V, Iba K, Somerville C. 1994. Cloning of a temperature-regulated gene encoding a chloroplast $\omega-3$ desaturase from Arabidopsis thaliana. Plant Physiol 106: 1615-1621.

Peer) reviewing PDF | (2020:06:49637:6:0:NEW 12 Jan 2021) 
Guo X, Liu D, Chong K. 2018. Cold signaling in plants: Insights into mechanisms and regulation. J Integr Plant Biol 60: 745-756.

Haas BJ, Papanicolaou A, Yassour M, Grabherr M, Blood PD, Bowden J, Couger MB, Eccles D, Li B, Lieber M, Macmanes MD, Ott M, Orvis J, Pochet N, Strozzi F, Weeks N, Westerman R, William T, Dewey CN, Henschel R, Leduc RD, Friedman N, Regev A. 2013. De novo transcript sequence Reconstruction from RNA-seq using the Trinity platform for reference Generation and analysis. Nat Protoc 8: 1494-1512.

Hara M, Terashima S, Fukaya T, Kuboi T. 2003. Enhancement of cold tolerance and inhibition of lipid peroxidation by citrus dehydrin in transgenic tobacco. Planta 217: 290-298.

He J, Yang Z, Hu B, Ji X, Wei Y, Lin L, Zhang Q. 2015. Correlation of polyunsaturated fatty acids with the cold adaptation of Rhodotorula glutinis. Yeast 32: 683-690.

Hendrickson L, Vlcková A, Selstam E, Huner N, Oquist G, Hurry V. 2006. Cold acclimation of the Arabidopsis dgd1 mutant results in recovery from photosystem I-limited photosynthesis. FEBS Lett 580: 4959-4968.

Kim SI, Tai TH. 2011. Evaluation of seedling cold tolerance in rice cultivars: a comparison of visual ratings and quantitative indicators of physiological changes. Euphytica 178: 437-447.

Kim SH, Kim HS, Bahk S, An J, Yoo Y, Jy K, Chung WS. 2017. Phosphorylation of the transcriptional repressor MYB15 by mitogen-activated protein kinase 6 is required for freezing tolerance in Arabidopsis. Nucleic Acids Res 45: 6613-6627.

Krasensky J, Jonak C. 2012. Drought, salt, and temperature stress-induced metabolic rearrangements and regulatory networks. J Exp Bot 63: 1593-1608.

Krishnan N, Dickman MB, Becker DF. 2008. Proline modulates the intracellular redox environment and protects mammalian cells against oxidative stress. Free Radic Biol Med 44: 671-681.

Li H, Ding YL, Shi YT, Zhang XY, Zhang SQ, Gong ZZ, Yang SH. 2017. MPK3- and MPK6-mediated ICE1 phosphorylation negatively regulates ICE1 stability and freezing tolerance in Arabidopsis. Dev Cell 43: $630-642$.

Li B, Dewey CN. 2011. RSEM: accurate transcript quantification from RNA-Seq data with or without a reference genome. BMC Bioinformatics 12: 323.

Liu Q, Kasuga M, Sakuma Y, Abe H, Miura S, Yamaguchi-shinozaki K, Shinozaki K. 1998. Two transcription factors, DREB1 and DREB2, with an EREBP/AP2 DNA binding domain separate two cellular signal transduction pathways in drought- and low-temperature-responsive gene expression, respectively, in Arabidopsis. Plant Cell 10: 1391-1406.

Liu S, Li AD, Chen CH, Cao GJ, Zhang LM, Guo CY, Xu M. 2017A. DE NOVO transcriptome sequencing in Passiflora edulis SIMS to identify genes and signaling pathways involved in cold tolerance. Forests $\mathbf{8}$ : 435.

Liu Z, Jia Y, Ding Y, Shi Y, Li Z, Guo Y, Gong Z, Yang S. 2017B. Plasma membrane CRPK1-mediated phosphorylation of 14-3-3 proteins induces their nuclear import to fine-tune CBF signaling during cold response. Mol Cell 66: 117-128.

Liu J, Shi Y, Yang S. 2018A. Insights into the regulation of C-repeat binding factors in plant cold signaling. J Integr Plant Biol 60: 780-795.

Liu Y, Xu C, Zhu Y, Zhang L, Chen T, Zhou F, Chen H, Lin Y. 2018B. The calcium-dependent kinase 
OsCPK24 functions in cold stress responses in rice. J Integr Plant Biol 60: 173-188.

Liu X, Fu L, Qin P, Sun Y, Liu J, Wang X. 2019. Overexpression of the wheat trehalose 6-phosphate synthase 11 gene enhances cold tolerance in Arabidopsis thaliana. Gene 710: 210-217.

Livak KJ, Schmittgen TD. 2001. Analysis of relative gene expression data using real-time quantitative PCR and the 2(-Delta Delta C(T)) Method. Methods 25: 402-408.

Love MI, Huber W, Anders S. 2014. Moderated estimation of fold change and dispersion for RNA-seq data with DESeq2. Genome Biol 15: 550.

Ma Y, Dai X, Xu Y, Luo W, Zheng X, Zeng D, Pan Y, Lin X, Liu H, Zhang D, Xiao J, Guo X, Xu S, Niu Y, Jin J, Zhang H, Xu X, Li L, Wang W, Qian Q, Ge S, Chong K. 2015. COLD1 confers chilling tolerance in rice. Cell 160: 1209-1221.

Mann m JO. 2003. Proteomic analysis of post-translational modifications. Nat Biotechnol 21: 255-261.

Saijo Y, Hata S, Kyozuka J, Shimamoto K, Izui K. 2000. Over-expression of a single Ca2+-dependent protein kinase confers both cold and salt/drought tolerance on rice plants. Plant J 23: 319-327.

Salzberg SL, Langmead B. 2012. AST gapped-read alignment with bowtie 2. Nat Methods 9: 357-359.

Seong es BS, Cho hs CD. 2007. Induction of enhanced tolerance to cold stress and disease by overexpression of the pepper CAPIF1 gene in tomato. Physiol Plant 129: 555-566.

Shi J, Cao Y, Fan X, Li M, Wang Y, Ming F. 2012. A rice microsomal delta-12 fatty acid desaturase can enhance resistance to cold stress in yeast and Oryza sativa. Mol Breeding 29: 743-757.

Shi Y, Ding Y, Yang S. 2018. Molecular regulation of CBF signaling in cold acclimation. Trends Plant Sci 23: 623-637.

Soto T, Beltrán FF, Paredes V, Madrid M, Millar JB, Vicente-soler J, Cansado J, Gacto M. 2002. Cold induces stress-activated protein kinase-mediated response in the fission yeast Schizosaccharomyces pombe. Eur J Biochem 269: 5056-5065.

Stockinger EJ, Gilmour SJ, Thomashow MF. 1997. Arabidopsis thaliana CBF1 encodes an AP2 domaincontaining transcriptional activator that binds to the C-repeat/DRE, a cis-acting DNA regulatory element that stimulates transcription in response to low temperature and water deficit. Proc Natl Acad Sci U S A 94: 1035-1040.

Su G, Morris JH, Demchak B, Bader GD. 2014. Biological network exploration with Cytoscape 3. Curr Protoc Bioinformatics 8: 8.13.1-8.1.

Teige M, Scheikl E, Eulgem T, Dóczi R, Ichimura K, Shinozaki K, Dangl JL, Hirt H. 2004. The MKK2 pathway mediates cold and salt stress signaling in Arabidopsis. Mol Cell 15: 141-152.

Upchurch RG. 2008. Fatty acid unsaturation, mobilization, and regulation in the response of plants to stress. Biotechnol Lett 30: 967-977.

Vogel JT, Zarka DG, Van buskirk HA, Fowler SG, Thomashow MF. 2005. Roles of the CBF2 and ZAT12 transcription factors in configuring the low temperature transcriptome of Arabidopsis. Plant J 41: 195-211.

Wang DZ, Jin YN, Ding XH, Wang WJ, Zhai SS, Bai LP, Guo ZF. 2017. Gene regulation and signal transduction in the ICE-CBF-COR signaling pathway during cold stress in plants. Biochemistry (Mosc) 82: $1103-1117$.

Wang H, Tang J, Liu J, Hu J, Liu J, Chen Y, Cai Z, Wang X. 2018. Abscisic acid signaling inhibits brassinosteroid signaling through dampening the dephosphorylation of BIN2 by ABI1 and ABI2. Mol Plant 11: 315-325. 
Wang X, Ding Y, Li Z, Shi Y, Wang J, Hua J, Gong Z, Zhou JM, Yang S. 2019. PUB25 and PUB26 promote plant freezing tolerance by degrading the cold signaling negative regulator MYB15. Dev Cell 51: 222-235.

Webb MS, Uemura M, Steponkus PL. 1994. A comparison of freezing injury in oat and rye: two cereals at the extremes of freezing tolerance. Plant Physiol 104: 467-478.

Wu J, Zhang Y, Yin L, Qu J, Lu J. 2014. Linkage of cold acclimation and disease resistance through plantpathogen interaction pathway in Vitis amurensis grapevine. Funct Integr Genomics 14: 741-755.

Wu Y, Tian Q, Huang W, Liu J, Xia X, Yang X, Mou H. 2020. Identification and evaluation of reference genes for quantitative real-time PCR analysis in Passiflora edulis under stem rot condition. Mol Biol Rep 47: 2951-2962.

Xie G, Kato H, Imai R. 2012. Biochemical identification of the OsMKK6-OsMPK3 signalling pathway for chilling stress tolerance in rice. Biochem J 443: 95-102.

Yadav SK. 2010. Cold stress tolerance mechanisms in plants. Agron Sustain Dev 30: 605-620.

Yamori W, Hikosaka K, Way DA. 2014. Temperature response of photosynthesis in C3, C4, and CAM plants: temperature acclimation and temperature adaptation. Photosynth Res 119: 101-117.

Ye K, Li H, Ding Y, Shi Y, Song C, Gong Z, Yang S. 2019. BRASSINOSTEROID-INSENSITIVE2 negatively regulates the stability of transcription factor ICE1 in response to cold stress in Arabidopsis. Plant Cell 31: 2682-2696.

Yuan P, Du L, Poovaiah BW. 2018A. Ca2+/Calmodulin-dependent AtSR1/CAMTA3 plays critical roles in balancing plant growth and immunity. Int J Mol Sci 19: E1764.

Yuan P, Yang T, Poovaiah BW. 2018B. Calcium signaling-mediated plant response to cold stress. Int J Mol Sci 19: E3896.

Yue C, Cao HL, Wang L, Zhou YH, Huang YT, Hao XY, Yc W, Wang B, Yang YJ, Wang XC. 2015. Effects of cold acclimation on sugar metabolism and sugar-related gene expression in tea plant during the winter season. Plant Mol Biol 88: 591-608.

Zhang J, Liu H, Sun J, Li B, Zhu Q, Chen S, Zhang H. 2012. Arabidopsis fatty acid desaturase FAD2 is required for salt tolerance during seed germination and early seedling growth. PLoS One 7: e30355.

Zhang Z, Li J, Pan Y, Li J, Zhou L, Shi H, Zeng Y, Guo H, Yang S, Zheng W, Yu J, Sun X, Li G, Ding Y, Ma L, Shen S, Dai L, Zhang H, Yang S, Guo Y, Li Z. 2017A. Natural variation in CTB4a enhances rice adaptation to cold habitats. Nat Commun 8: 14788.

Zhang Z, Li J, Li F, Liu H, Yang W, Chong K, Xu Y. 2017B. OsMAPK3 phosphorylates OsbHLH002/OsICE1 and inhibits its ubiquitination to activate OsTPP1 and enhances rice chilling tolerance. Dev Cell 43: 731-745.

Zhang F, Lu K, Gu Y, Zhang L, Li W, Li Z. 2020. Effects of low-temperature stress and brassinolide application on the photosynthesis and leaf structure of tung tree seedlings. Front Plant Sci 10: 1767.

Zhao C, Wang P, Si T, Hsu CC, Wang L, Zayed O, Yu Z, Zhu Y, Dong J, Tao W, Zhu JK. 2017A. MAP kinase cascades regulate the cold response by modulating ICE1 protein stability. Dev Cell 43: 618-629.

Zhao C, Wang P, Si T, Hsu CC, Wang L, Zayed O, Yu Z, Zhu Y, Dong J, Tao W, Zhu JK. 2017B. Map kinase cascades regulate the cold response by modulating ICE1 protein stability. Dev Cell 43: 618-629. 


\section{Table $\mathbf{1}$ (on next page)}

Statistical results of transcriptome sequencing 
1

2

Table 1 Statistical results of transcriptome sequencing

\begin{tabular}{ccccc}
\hline Sample & Reads number & Total base $(\mathrm{bp})$ & Q30 (\%) & GC content (\%) \\
\hline HJGA1 & 46599672 & 6989950800 & 93.02 & 45.36 \\
HJGA2 & 44522400 & 6678360000 & 93.08 & 44.49 \\
HJGA3 & 46642622 & 6996393300 & 93.23 & 45.31 \\
TN1A1 & 47166760 & 7075014000 & 93.18 & 45.23 \\
TN1A2 & 43505726 & 6525858900 & 93.31 & 44.33 \\
TN1A3 & 45058566 & 6758784900 & 93.16 & 43.83 \\
HJGB1 & 44758052 & 6713707800 & 93.34 & 43.83 \\
HJGB2 & 43843522 & 6576528300 & 93.43 & 44.70 \\
HJGB3 & 39087142 & 5863071300 & 93.14 & 43.37 \\
TN1B1 & 49306634 & 7395995100 & 93.31 & 45.51 \\
TN1B2 & 44267228 & 6640084200 & 93.17 & 44.87 \\
TN1B3 & 39177250 & 5876587500 & 93.27 & 44.89 \\
\hline
\end{tabular}

3 
Table 2 (on next page)

Sequencing data mapped to unigene set 
1

2

Table 2 Sequencing data mapped to unigene set

\begin{tabular}{|c|c|c|c|c|c|}
\hline Sample & Pair reads & $\begin{array}{c}\text { Aligned concordantly } 0 \\
\text { times }\end{array}$ & $\begin{array}{c}\text { Aligned concordantly exactly } 1 \\
\text { time }\end{array}$ & Aligned concordantly $>1$ times & Total alignment ratio (\%) \\
\hline HJGA1 & 23299836 & 6183910 & 15486803 & 1629123 & 83.19 \\
\hline HJGA2 & 22261200 & 6461646 & 14313504 & 1486050 & 81.73 \\
\hline HJGA3 & 23321311 & 7002139 & 14768806 & 1550366 & 81.04 \\
\hline TN1A1 & 23583380 & 8597970 & 13025983 & 1959427 & 75.69 \\
\hline TN1A2 & 21752863 & 7919003 & 11974898 & 1858962 & 75.79 \\
\hline TN1A3 & 22529283 & 7817813 & 12764435 & 1947035 & 77.11 \\
\hline HJGB1 & 22379026 & 6946292 & 14131411 & 1301323 & 78.91 \\
\hline HJGB2 & 21921761 & 6859052 & 13821399 & 1241310 & 78.45 \\
\hline HJGB3 & 19543571 & 5267390 & 12862365 & 1413816 & 81.72 \\
\hline TN1B1 & 24653317 & 9000336 & 13737412 & 1915569 & 74.21 \\
\hline TN1B2 & 22133614 & 8908390 & 11548761 & 1676463 & 70.83 \\
\hline TN1B3 & 19588625 & 6676080 & 11320050 & 1592495 & 75.95 \\
\hline
\end{tabular}

3 
Table 3 (on next page)

Unigenes were annotated to 7 databases 
1

2

Table 3 Unigenes were annotated to 7 databases

\begin{tabular}{ccc}
\hline Database & Annotated number & Annotated ratio (\%) \\
\hline GO & 17123 & 36.16 \\
KEGG & 16086 & 33.97 \\
KOG & 23164 & 48.92 \\
NR & 46369 & 97.92 \\
Pfam & 29091 & 61.43 \\
Swiss-Prot & 33337 & 70.40 \\
TrEMBL & 46323 & 97.82 \\
Total & 47353 & 100 \\
\hline
\end{tabular}

3 


\section{Figure 1}

Annotation of passion fruit transcriptome

(A) GO function classification diagram of unigenes. The x-axis indicates the secondary classification terms of $\mathrm{GO}$; the $\mathrm{y}$-axis indicates the number of unigenes in this secondary classification out of the total annotated unigenes. (B) KOG functional annotation distribution of unigenes. The $x$-axis indicates the nuber unigens; the $y$-axis indicates the name of 25 groups. (C) KEGG classification of unigenes. The $x$-axis indicates the number of unigenes in the pathway; the $y$-axis indicates KEGG pathways. 


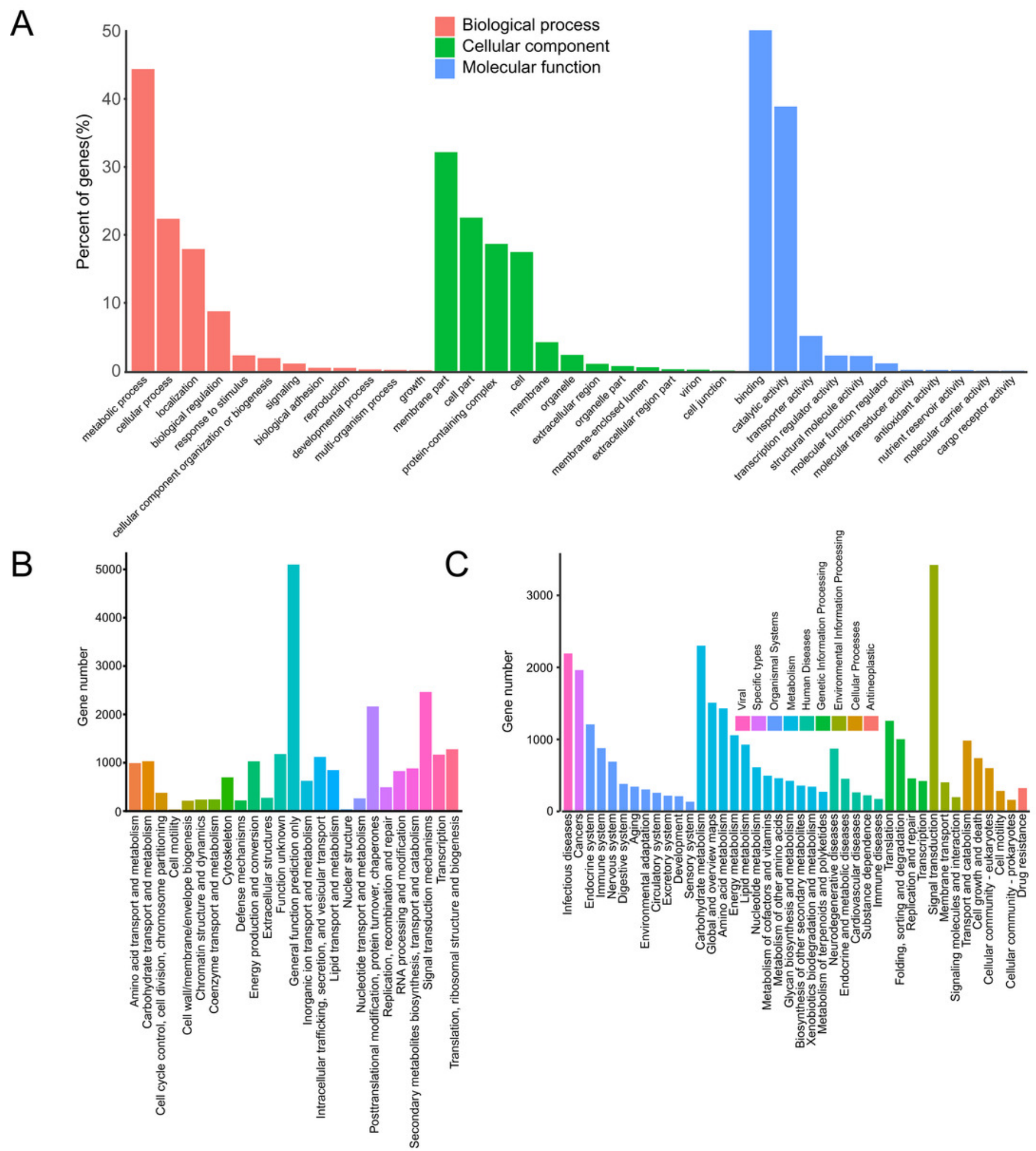




\section{Figure 2}

Analysis of DEGs at two stages.

(A) DEGs identified between HJG and TN1. (B) A1 vs A2; (C) B1 vs B2. Red indicates that the gene is highly expressed in the sample; blue indicates lower expression, and the number label under the color bar at the upper left is the specific trend of the change of expression. The left is a dendrogram of gene clustering, and below is the name of the samples. Figure 3 KEGG pathway enrichment of DEGs. (A) A1 vs A2. (B) B1 vs B2. The left is the name of pathways, and below is the enrichment factor. The size of the dots indicate the number of genes in this pathway, and the color of the dots corresponds to different -log10(correct $p$ value) ranges.

A

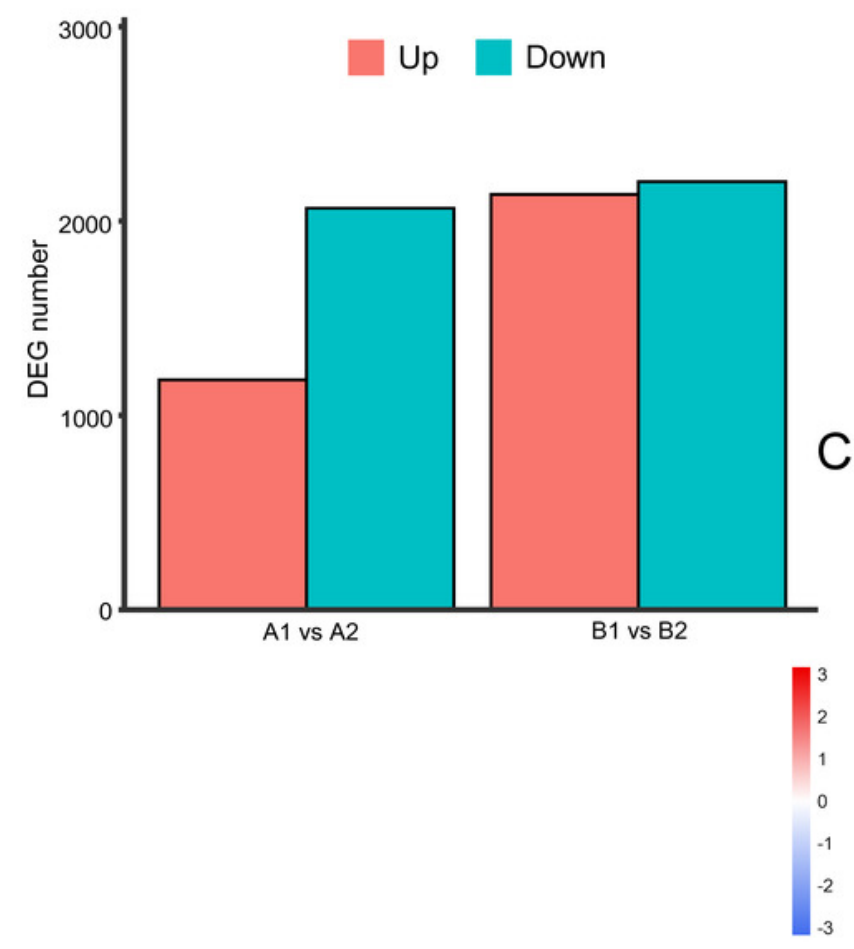

B

C
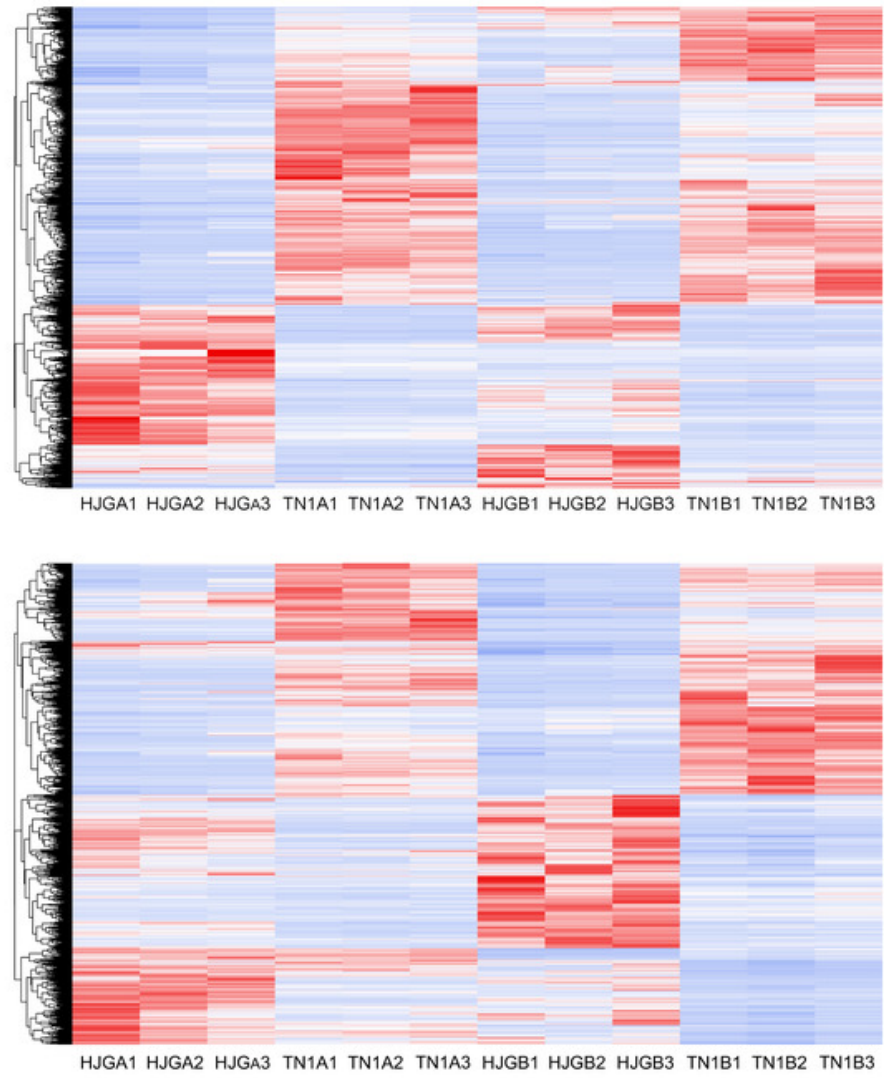
Figure 3

KEGG pathway enrichment of DEGs.

(A) $A 1$ vs $A 2$. (B) B1 vs B2. The left is the name of pathways, and below is the enrichment factor. The size of the dots indicate the number of genes in this pathway, and the color of the dots corresponds to different $-\log 10$ (correct $p$ value) ranges.

A

Systemic lupus erythematosus. Legionellosis Bile secretion.

Peroxisome. Plant hormone signalar transport PPAR signaling pathway. Biosynthesis of amino acids. Fatty acid metabolism Carbon metabolism. Biosynthesis of unsaturated fatty acids. Tropane, piperidine and pyridine alkaloid biosynthesis Phenylpropanoid biosynthesis . Sulfur metabolism.
Penoid biosynthesis. Sesquiterpenoid and triterpenoid biosynthesis-
Zeatin biosynthesis. Monoterpenoid biosynthesis Riboflavin metabolism. Glyoxylate and dicarboxylate metabolism. Glycosphingolipid biosynthesis - ganglio series alpha-Linolenic acid metabolism. Glycosaminoglycan degradation. Starch and sucrose metabolism. Glutathione metabolism. Cyanoamino acid metabolism . Cysteine and methionine metabolism. Photosynthesis - antenna proteins.
Pats antenna proteins
Photosynthesis. Steroid biosynthesis

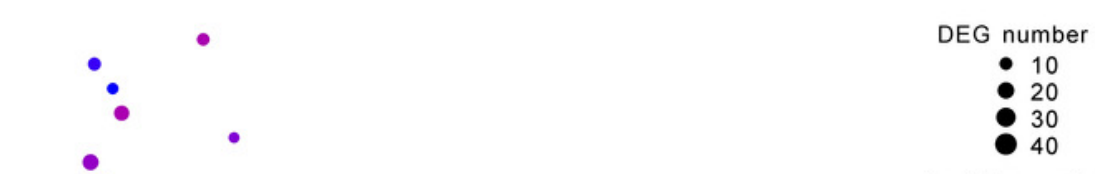

number

- 10

40

- $\log 10$ (correct p-value)
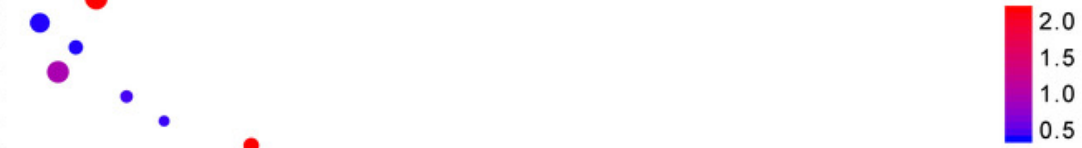

B

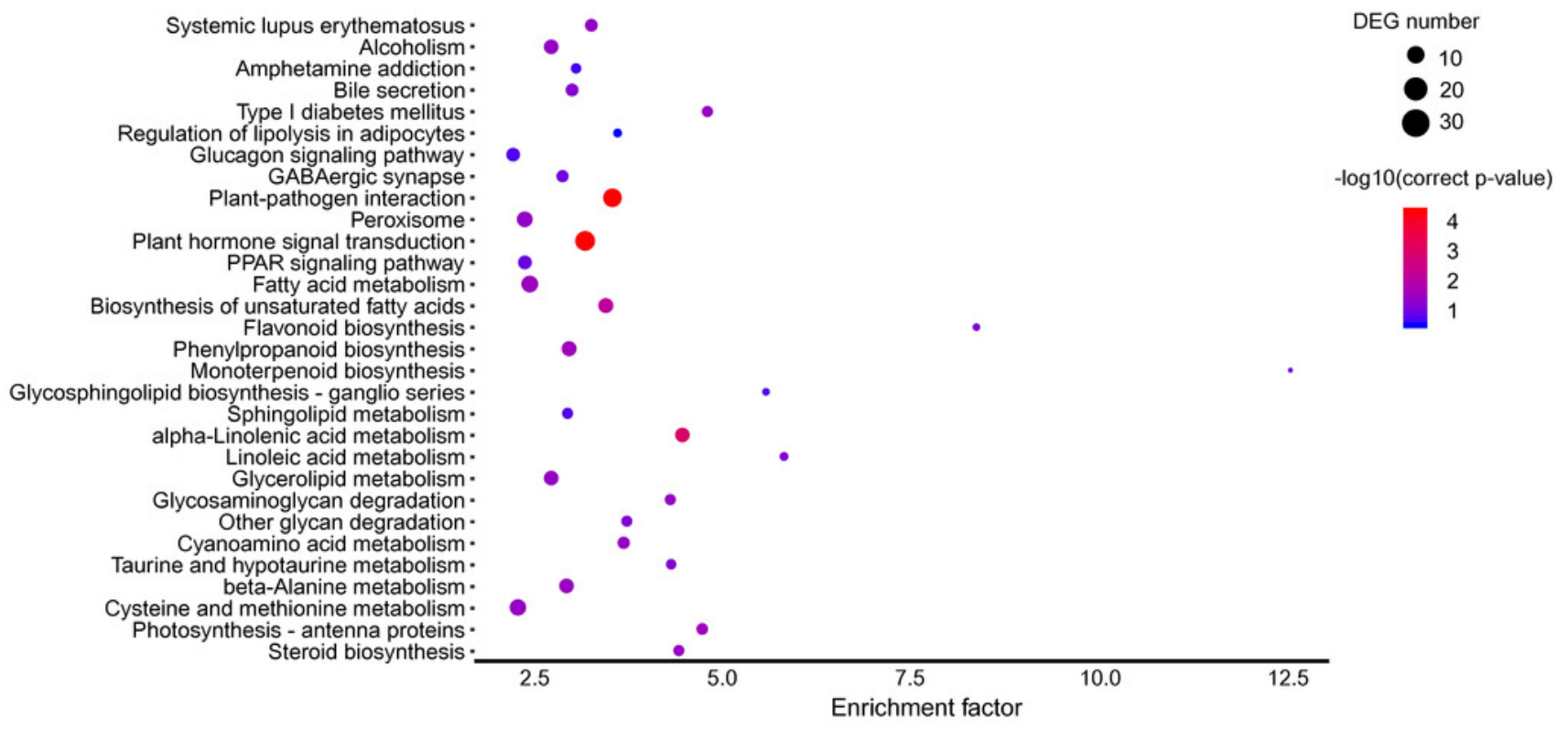




\section{Figure 4}

Thr heat map of DEGs at two stages.

(A) A1 vs A2. (B) B1 vs B2. The below is the name of the samples. Red indicates that the gene is highly expressed in the sample; blue indicates lower expression, and the number label under the color bar at the upper left is the specific trend of the change of expression.

A

B

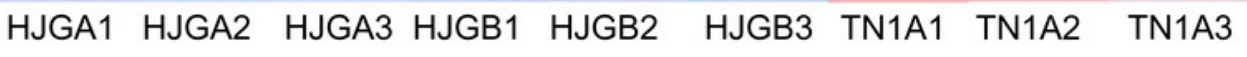

TN1B1 TN1B2 TN1B3

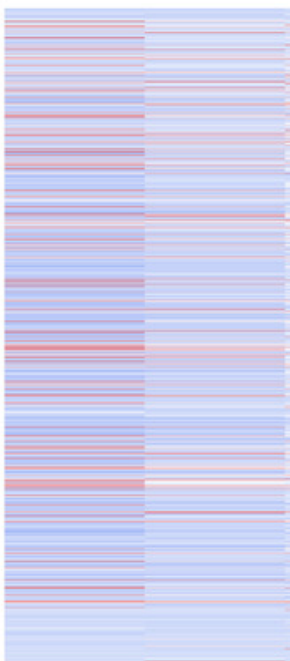

HJGA1 HJGA2 HJGA3 HJGB1 HJGB2 HJGB3 TN1A1 TN1A2 TN1A3 TN1B1 TN1B2 TN1B3 
Figure 5

KEGG pathway enrichment in two co-expression modules.

(A) Brown module. (B) Yellow module. The x-axis indicates the enrichment factor; the y-axis indicates the name of pathways. The size of the dots indicate the number of genes in this pathway, and the color of the dots corresponds to different - $\log 10$ (correct $p$ value) ranges.

A

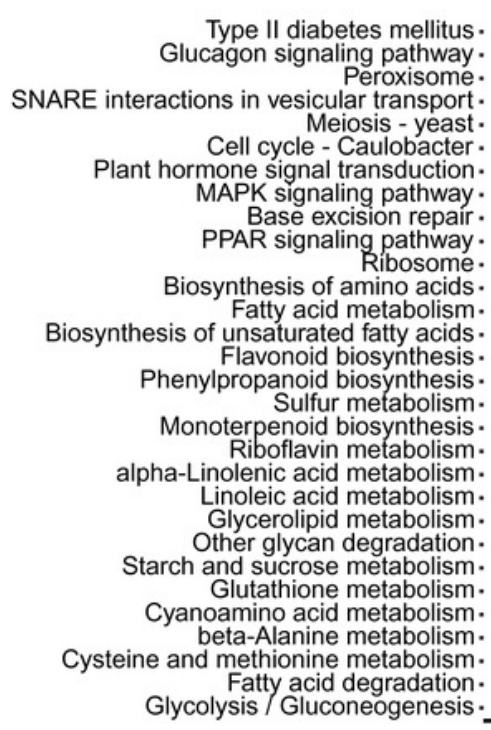
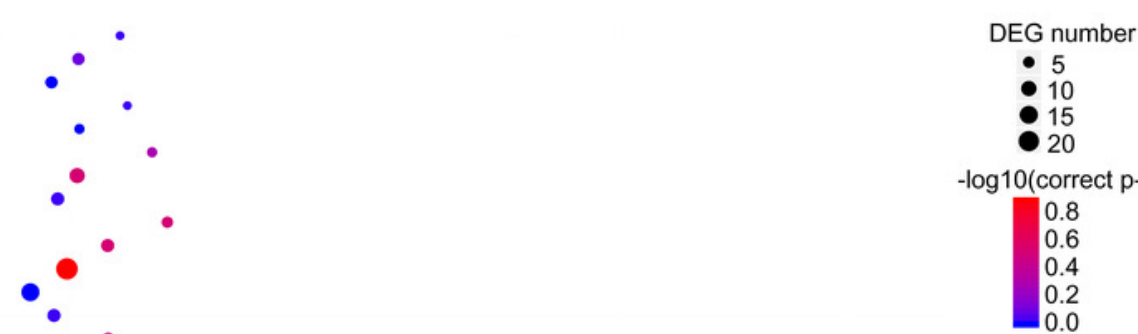

- $\log 10$ (correct p-value)

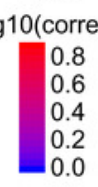

B

NA:NA.
Systemic lupus erythematosus. Legionellosis. Bile secretion. Type I diabetes mellitus. Glucagon signaling pathway Circadian rhythm - plant.

Hippo signaling pathway - fly. SNARE interactions in vesicular transportPlant hormone signal transduction. Flagellar assembly. Two-component system.
ABC transporters. $A B C$ transporters

Cationic antimicrobial peptide (CAMP) resistance.

Biosynthesis of amino acids
Isoflavonoid biosynthesis Limonene and pinene degradation. Porphyrin and chlorophyll metabolism. Folate biosynthesis Thiamine metabolism. Chloroalkane and chloroalkene degradation Starch and sucrose metabolism. beta-Alanine metabolism Phenylalanine, tyrosine and tryptophan biosynthesis. Tryptophan metabolism.
. Histidine metabolism

Arginine and proline metabolism. Lysine degradation. Cysteine and methionine metabolism. Pentose and glucuronate interconversions .

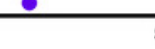
10 Enrichment factor

DEG number

- 25

- 50

? 75

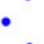

$\bullet$

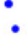

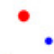

$\because$

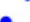

-log10(correct_p_value)

0.9

0.6

0.3

0.0 
Figure 6

Gene coexpression network related to cold stress.

(A) Gene co-expression network related to cold stress in brown module. (B) Gene coexpression network related to cold stress in yellow module. Red dots represent the hube gene belonging to the co-expression network.

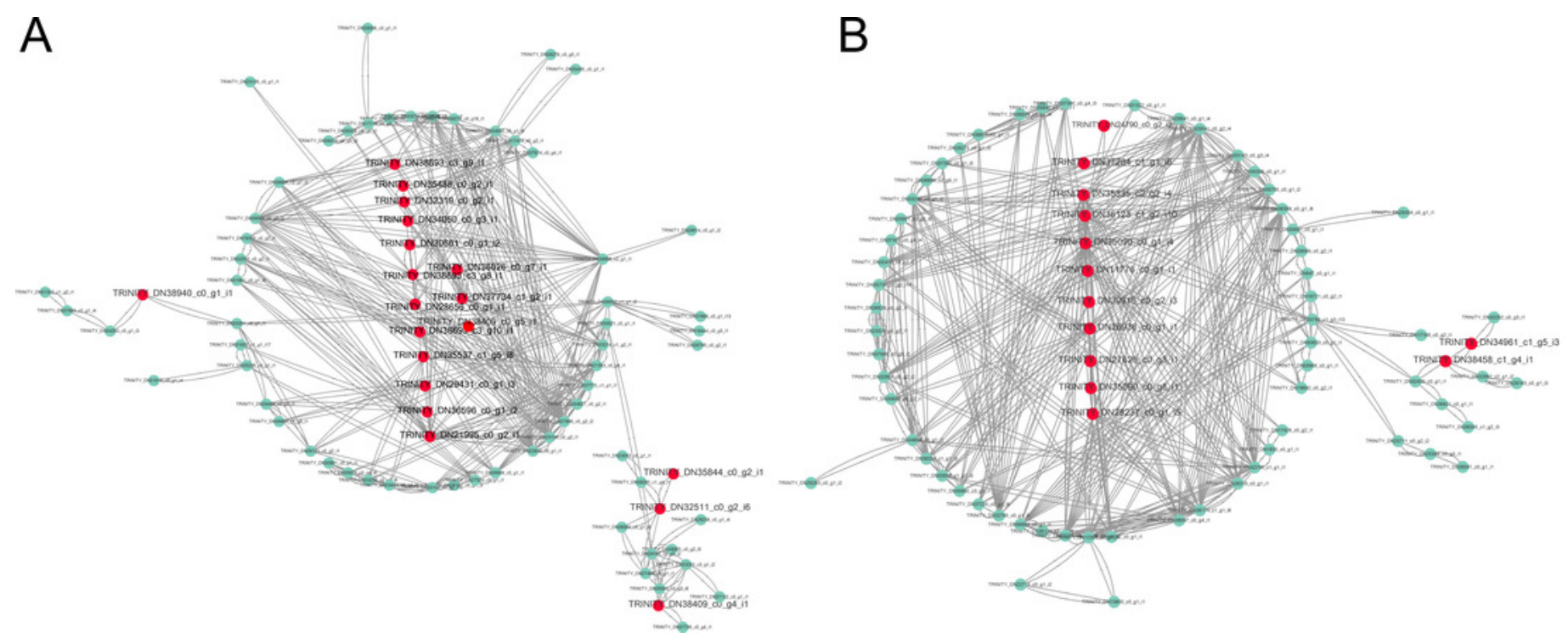


Figure 7

Cold acclimation related genes were validated by RT-qPCR.

The blocks indicate the samples of HJG and TN1 using in RT-qPCR and RNA-seq under cold stress condition. Bars indicate standard deviations of three biological repetitions.

A

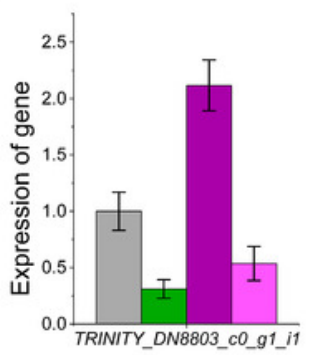

$\mathrm{F}$

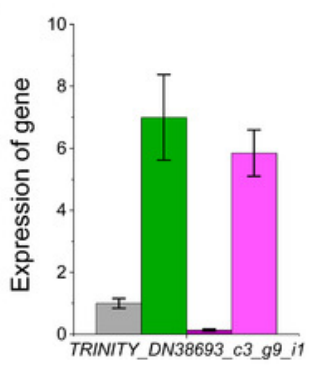

$\mathrm{K}$

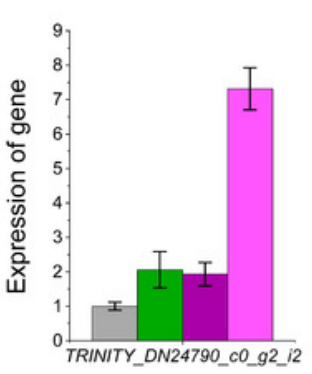

B

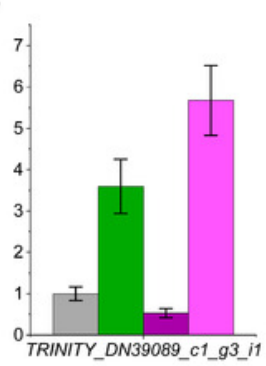

G

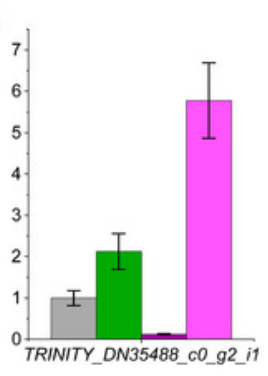

$\mathrm{L}_{250}$

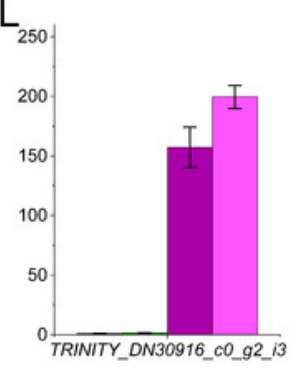

C

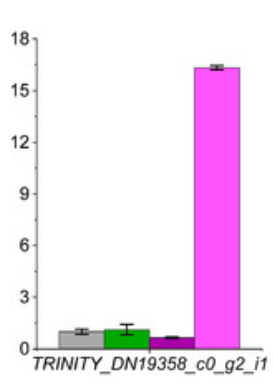

$\mathrm{H}$

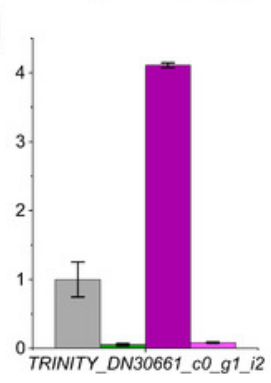

$\mathrm{M}$

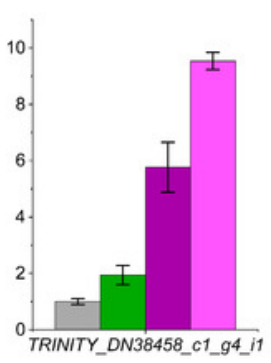

D
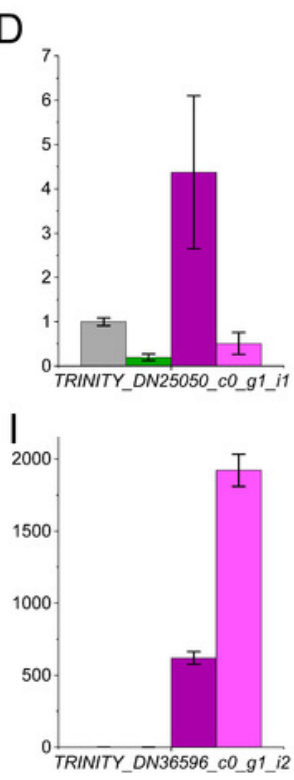

$\mathrm{N}_{1.4}$

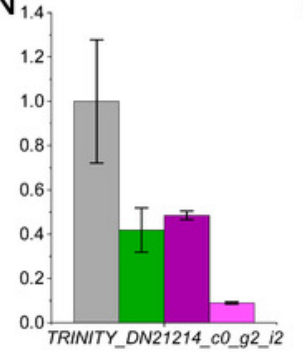

E

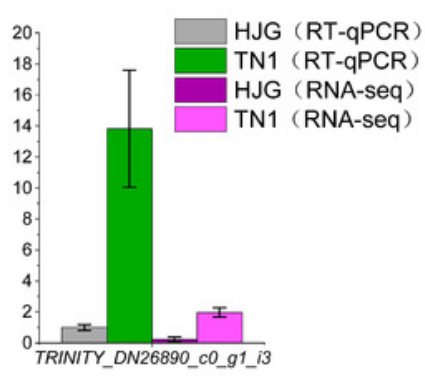

$\mathrm{J}_{16}$

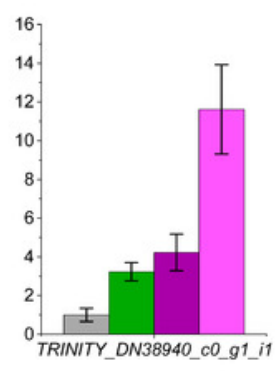

$\mathrm{O}$

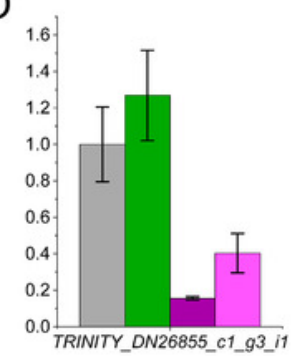

